\title{
ON NON-EXISTENCE OF GLOBAL SOLUTIONS TO A CLASS OF STOCHASTIC HEAT EQUATIONS
}

\author{
MOHAMMUD FOONDUN AND RANA D. PARSHAD
}

(Communicated by Mark M. Meerschaert)

\begin{abstract}
We consider nonlinear parabolic SPDEs of the form $\partial_{t} u=-$ $(-\Delta)^{\alpha / 2} u+b(u)+\sigma(u) \dot{w}$, where $\dot{w}$ denotes space-time white noise. The functions $b$ and $\sigma$ are both locally Lipschitz continuous. Under some suitable conditions on the parameters of this SPDE, we show that the above equation has no random-field solution. This complements recent works of Khoshnevisan and his coauthors.
\end{abstract}

\section{INTRODUCTION}

In [5], 6] and [3], Khoshnevisan and his coauthors initiated a research program, where they systematically studied the large time behavior of stochastic equations of the following type

$$
\begin{aligned}
\partial_{t} u(t, x) & =\mathcal{L} u(t, x)+\sigma(u(t, x)) \dot{F}(t, x), \\
u(0, x) & =u_{0}(x),
\end{aligned}
$$

where $\mathcal{L}$ is the generator of a Lévy process, $\sigma$ is a globally Lipschitz function, $\dot{F}$ is a noisy perturbation, and $u_{0}(x)$ is specified initial data satisfying certain suitable conditions. Among other things, the authors studied the upper $p$-th moment Liapounov exponent $\bar{\gamma}(p)$ defined as

$$
\bar{\gamma}(p):=\limsup _{t \rightarrow \infty} \frac{1}{t} \ln \mathrm{E}\left(|u(t, x)|^{p}\right) \quad \text { for all } \quad p \in(0, \infty)
$$

A motivation for this study comes from the Parabolic Anderson model and the phenomenon of intermittency which this model exhibits. Instead of going into details about this phenomenon, we refer the reader to 2] for details and references. One of the main results in the above works, is the existence and strict positivity of those upper Liapounov exponents. A common assumption in all the works mentioned above, is that the operator $\mathcal{L}$ models regular diffusion so one considers, say, the Laplace operator. Another important assumption is that the multiplicative non-linearity $\sigma$ is assumed globally Lipschitz. Indeed, this is required to ensure global existence of solutions. In this short note, we consider a subclass of (1.1), where we consider a fractional Laplacian operator instead of the diffusion process,

Received by the editors August 22, 2012 and, in revised form, September 21, 2012 and April 21, 2014.

2010 Mathematics Subject Classification. Primary 60H15; Secondary 82B44.

Key words and phrases. Stochastic partial differential equations, stable processes, Liapounov exponents, weak intermittence. 
and we drop the global Lipschitz property of $\sigma$. More precisely we look at the following equation:

$$
\begin{aligned}
\partial_{t} u(t, x) & =-(-\Delta)^{\alpha / 2} u(t, x)+b(u(t, x))+\sigma(u(t, x)) \dot{w}(t, x), \\
u(0, x) & =u_{0}(x),
\end{aligned}
$$

where $\{\dot{w}(t, x)\}_{t \geq 0, x \in \mathbf{R}}$ denotes space-time white noise. $-(-\Delta)^{\alpha / 2}$ is the fractional Laplacian; the $L^{2}$-generator of a symmetric stable process of order $\alpha$, so that $\operatorname{E} \exp \left(i \xi \cdot X_{t}\right)=\exp \left(-t|\xi|^{\alpha}\right)$. We will follow Walsh [16] and interpret the above as an Itô-type stochastic PDE. The functions $b$ and $\sigma$ are assumed to be positive and locally Lipschitz. We will be interested in random field solution in the sense of Dalang [4] and in finite energy solution. We will define these notions with more precision later, but would like to mention that a standard assumption in this theory is that

$$
\Upsilon(\beta):=\frac{1}{2 \pi} \int_{-\infty}^{\infty} \frac{\mathrm{d} \xi}{\beta+2|\xi|^{\alpha}}<\infty \quad \text { for all } \beta>0 ;
$$

confer with [5] and [4. This implies that $\alpha \in(1,2]$, a condition which will be enforced throughout this paper. We will also assume the initial data $u_{0}$ is a positive bounded measurable function satisfying the following lower bound:

$$
\inf _{x \in \mathbf{R}} u_{0}(x) \geq \kappa,
$$

where $\kappa$ is a positive constant.

There are many physical motivations to consider a fractional Laplacian operator. These operators appear in many models in non-Newtonian fluids, in models of viscoelasticity such as Kelvin-Voigt models, various heat transfer processes in fractal and disordered media, and models of fluid flow and acoustic propogation in porous media. Interestingly, they have also recently been applied to pricing derivative securities in financial markets. See [1,7,8, for details on a number of the aforementioned applications. The essential feature behind many of these models is that they aim to capture the nonlinear relation between stress and strain, in nonNewtonian or viscoelastic models per se. Or perhaps, aim to model the disparity between scaling laws associated with diffusion, in euclidian geometries, and fractal geometries, such as certain porous media. Thus there are a plethora of reasons why one might consider investigating the asymptotic behavior of equations of the type (1.2). In particular, such investigations might help us glean information in many real physical scenarios, involving sub- or super-diffusions.

Before stating the main result of this paper, we fix some terminology. When we say that a function $f$ blows up in finite time, we mean that there exists a time $T$ such that for all $t \geq T, f(t)=\infty$. And when we say that a statement holds whenever a quantity $Q$ is large enough, we mean that the statement holds whenever $Q \geq Q_{0}$, where $Q_{0}$ is some positive constant.

Our result will hold under at least one of the following two conditions which are non-linear growth conditions on $\sigma$ and $b$ respectively.

Condition 1.1. There exist constants $\beta$ and $K_{1}>0$, both strictly positive such that

$$
\inf _{x \in R} \frac{\sigma(x)}{|x|^{1+\beta}} \geq K_{1}
$$


Condition 1.2. There exist constants $\gamma$ and $K_{2}$ both strictly positive such that

$$
\inf _{x \in R} \frac{b(x)}{|x|^{1+\gamma}} \geq K_{2} \text {. }
$$

We now define our notions of solutions with more precision. Let $p(t, \cdot)$ denote the transition density function of a symmetric stable process of order $\alpha$. The existence of this density is a well-known fact; see for instance 14. Set

$$
\mathcal{P}_{t} f(x):=\int_{-\infty}^{\infty} p(t, x-y) f(y) \mathrm{d} y .
$$

We can now follow the method of Walsh [16] to see that (1.2) admits a mild solution $u$ if and only if $u$ is a predictable process that satisfies

$$
\begin{aligned}
u(t, x) & =\mathcal{P}_{t} u_{0}(x)+\int_{-\infty}^{\infty} \int_{0}^{t} b(u(s, y)) p(t, y-x) \mathrm{d} s \mathrm{~d} y \\
& +\int_{-\infty}^{\infty} \int_{0}^{t} \sigma(u(s, y)) p(t-s, y-x) w(\mathrm{~d} s \mathrm{~d} y) .
\end{aligned}
$$

Following [4, we say that $u(t, x)$ is a random field solution to (1.2) if $u(t, x)$ defined above further satisfies

$$
\sup _{0<t \leq T-\infty<y<\infty} \sup _{-\infty} \mathrm{E}|u(t, x)|^{2}<\infty,
$$

for all $0<T<\infty$, and we say that $u(t, x)$ is a finite-energy solution to (1.2) if there exists some $\rho>0$ such that

$$
\int_{0}^{\infty} e^{-\rho t} \mathrm{E}|u(t, x)|^{2} \mathrm{~d} t<\infty \quad \text { for all } \quad x \in \mathbf{R} .
$$

We can now state our main result.

Theorem 1.3. (a) Assume that Condition 1.1 holds, then (1.2) has no finite energy solution.

(b) Suppose that Condition 1.2 is in force; then (1.2) has no random field solution.

A requirement for the existence of random field solution is that the second moment of the solution exists and is finite at each time. Our proof for non-existence will exploit the "acute non-linearity" of (1.2) and will show that if the second moment of the solution exists, then it has to blow up in finite time.

We will draw inspiration from blow-up problems in deterministic cases which is an area of intense study. The review articles of 9] and [10] contain a detailed exposition of the subject. A common approach in tackling these various blow-up problems is to consider a "right" quantity, which blows up in finite time, usually by exercising a differential inequality. The quantity at hand is usually linked to a certain "norm" of the solution. We will use a similar approach to prove our main result. One of the main difficulties here is to find this "right" quantity; see Proposition 3.1 .

We now briefly give an outline of the paper. In Section 2, we recall some facts about the heat kernel of the fractional Laplacian. In Section 3, we prove the main result. Finally in Section 4, we provide some concluding remarks. Throughout the paper, we use the letter $c$ with or without subscripts to denote a constant whose value is not important and may vary from place to place. And by $f(x) \asymp g(x)$, we mean that there exists a constant $K$ such that $\frac{1}{K} g(x) \leq f(x) \leq K g(x)$. 


\section{SOME AUXILIARY RESUlts}

The proof of our theorem will involve some bounds on the transition density function appearing in (1.4). Our first proposition recalls a few well-known properties which we will use to derive those necessary bounds. We do not present proofs but mention [14, where the reader can find lots of details about symmetric stable processes.

Proposition 2.1. The transition density $p(t, \cdot)$ of a strictly $\alpha$-stable process satisfies

(a)

$$
p(s t, x)=t^{-1 / \alpha} p\left(s, t^{-1 / \alpha} x\right) .
$$

(b) For t large enough such that $p(t, 0) \leq 1$ and $a>2$, we have

$$
p(t,(x-y) / a) \geq p(t, x) p(t, y) \quad \text { for all } \quad x \in \mathbf{R} .
$$

(c)

$$
p(t, x) \asymp t^{-1 / \alpha} \wedge \frac{t}{|x|^{1+\alpha}}
$$

As a first application of the above proposition, we have the following lemma.

Lemma 2.2. Suppose that $f(x) \not \equiv 0$. There exist positive constants $c$ and $t_{0}$ such that for all $t>0$,

$$
\int_{\mathbf{R}} \int_{\mathbf{R}} f(y) p\left(t_{0}, x-y\right) p(t, x) \mathrm{d} y \mathrm{~d} x \geq \frac{c}{\left(t_{0}+2^{\alpha} t\right)^{1 / \alpha}} .
$$

Proof. We begin by the following observation. For $t_{0}$ large enough, we have

$$
\begin{aligned}
p\left(t_{0}, x-y\right) & \geq p\left(t_{0}, 2 x\right) p\left(t_{0}, 2 y\right) \\
& \geq \frac{1}{2} p\left(t_{0} / 2^{\alpha}, x\right) p\left(t_{0}, 2 y\right) .
\end{aligned}
$$

We can therefore write

$$
\begin{aligned}
\int_{\mathbf{R}} \int_{\mathbf{R}} f(y) p\left(t_{0}, x-y\right) p(t, x) \mathrm{d} y \mathrm{~d} x & \geq \frac{1}{2} \int_{\mathbf{R}} \int_{\mathbf{R}} f(y) p\left(t_{0} / 2^{\alpha}, x\right) p\left(t_{0}, 2 y\right) \mathrm{d} y p(t, x) \mathrm{d} x \\
& =\frac{c_{1}}{2} \int_{\mathbf{R}} p\left(t_{0} / 2^{\alpha}, x\right) p(t, x) \mathrm{d} x \\
& =\frac{c}{\left(t_{0}+2^{\alpha} t\right)^{1 / \alpha}},
\end{aligned}
$$

where the constant $c$ obviously depends on $f$ and $t_{0}$.

Our next result concerns the blow-up of solutions to the class of ODE. We will use this to conclude the proof of one our main results.

Proposition 2.3. Consider the ordinary differential equation

$$
y^{\prime}(t) t^{b}=y(t)^{1+a}
$$

where $a, b>0$ with initial condition $y\left(t_{0}\right)$. If $b \leq 1$, the solution blows up for any positive initial condition $y\left(t_{0}\right)$. However, if $b>1$, then the solution blows up if and only if $y\left(t_{0}\right)$ is large enough. 
Proof. We begin by setting $z(t):=[y(t)]^{-a}$ so that $z^{\prime}(t)=-a t^{-b}$ which we can explicitly solve to find that

$$
z(t)= \begin{cases}z\left(t_{0}\right)-a \ln \left(t / t_{0}\right) & \text { if } b=1 \\ z\left(t_{0}\right)+\frac{a}{b-1}\left(t^{1-b}-t_{0}^{1-b}\right) & \text { if } b \neq 1\end{cases}
$$

Solving for the zeros of $z(t)$ finishes the proof.

We end this section by recalling Jensen's inequality which we will use frequently in the forthcoming section.

Lemma 2.4. Let $p(\mathrm{~d} x)$ be a probability measure on $\mathbf{R}$ and $u$ be a non-negative function. Then the following holds whenever $f$ is a convex function:

$$
f\left(\int_{R} u(x) p(\mathrm{~d} x)\right) \leq \int_{R} f(u(x)) p(\mathrm{~d} x) .
$$

\section{Proof of the MAIN RESUlt}

The proof of the main theorem hinges on the next proposition which is an $a$ priori result. That is, its statement implicitly assumes that the moment of the solution exists, at least up to a finite time. We need the following notation for the statement of this proposition.

Denote

$$
G(t):=\int_{\mathbf{R}} \mathrm{E}|u(t, y)| p(t, y) \mathrm{d} y,
$$

where $\{u(t, x)\}_{t \geq 0, x \in \mathbf{R}}$ is the solution to (1.2). We can then state and prove the following. Sugitani [15] was the main source of inspiration for its proof.

Proposition 3.1. Assume condition 1.2 holds; then the following statement holds. $G(t)$ blows up in finite time if and only if for all $x \in \mathbf{R}, \mathrm{E}|u(t, x)|$ blows up in finite time.

Proof. Note that it is enough to show that $\mathrm{E}|u(t, x)|=\infty$ whenever $G(t)=\infty$ for some large $t$. The other implication is easy. Using property (2.1), we can write

$$
p(t-s, x-y)=\left(\frac{s}{t-s}\right)^{1 / \alpha} p\left(s,\left(\frac{s}{t-s}\right)^{1 / \alpha}(x-y)\right) .
$$

Let $\tilde{c}_{\alpha}:=\frac{1}{2^{\alpha}+1}$. We can now use (2.2) to bound the right hand side of the above display as follows:

$$
p(t-s, x-y) \geq\left(\frac{s}{t-s}\right)^{1 / \alpha} p(s, x) p(s, y),
$$

for $t_{0} \leq s \leq \tilde{c}_{\alpha} t$, where $t_{0}$ is chosen large enough so that $p(s, 0) \leq 1$ for all $s \geq t_{0}$. From the mild formulation of the solution i.e. (1.5) and the positivity of the initial condition $u_{0}(x)$, we obtain

$$
\mathrm{E} \mid u(t, x))\left|\geq \int_{0}^{t} \int_{\mathbf{R}} \mathrm{E}\right| b(u(s, y)) \mid p(t-s, x-y) \mathrm{d} y \mathrm{~d} s .
$$

Since the integrand appearing in the right hand side of the above display is strictly positive, we have

$$
\mathrm{E} \mid u(t, x))\left|\geq \int_{t_{0}}^{\tilde{c}_{\alpha} t} \int_{\mathbf{R}} \mathrm{E}\right| b(u(s, y)) \mid p(t-s, x-y) \mathrm{d} y \mathrm{~d} s .
$$


Using the bound (3.2), and the lower bound on $b$ together with Jensen's inequality, we can write

$$
\begin{aligned}
\mathrm{E} \mid u(t, x)) \mid & \geq \int_{t_{0}}^{\tilde{c}_{\alpha} t}\left(\frac{s}{t-s}\right)^{1 / \alpha} p(s, x) \int_{\mathbf{R}} \mathrm{E}|b(u(s, y))| p(s, y) \mathrm{d} y \mathrm{~d} s \\
& \geq c_{1} \int_{t_{0}}^{\tilde{c}_{\alpha} t}\left(\frac{s}{t-s}\right)^{1 / \alpha} p(s, x) \int_{\mathbf{R}}(\mathrm{E}|u(s, y)|)^{1+\gamma} p(s, y) \mathrm{d} y \mathrm{~d} s \\
& \geq c_{1} \int_{t_{0}}^{\tilde{c}_{\alpha} t}\left(\frac{s}{t-s}\right)^{1 / \alpha} p(s, x)\left(\int_{\mathbf{R}} \mathrm{E}|u(s, y)| p(s, y) \mathrm{d} y\right)^{1+\gamma} \mathrm{d} s .
\end{aligned}
$$

The result now follows from the above inequality and the strict positivity of the transition function.

We are now ready to prove our main result, Theorem 1.3 .

Proof. The proof of the first part of the theorem will be by contradiction. We will assume that there is a finite-energy solution and will come up with a contradiction. We start with (1.5) and bearing in mind the positivity of $b$, we take second moment

$$
\begin{aligned}
\mathrm{E}|u(t, x)|^{2} & \geq\left|\int_{\mathbf{R}} u_{0}(y) p(t, x-y) \mathrm{d} y\right|^{2} \\
& +\int_{\mathbf{R}} \int_{0}^{t} \mathrm{E}|\sigma(u(s, y))|^{2} p^{2}(t-s, x-y) \mathrm{d} s \mathrm{~d} y \\
& :=I_{1}+I_{2} .
\end{aligned}
$$

We bound the $I_{1}$ first as this is straightforward. The lower bound on the initial condition yields

$$
\int_{\mathbf{R}} u_{0}(y) p(t, x-y) \mathrm{d} y \geq \kappa
$$

Hence, we have

$$
I_{1} \geq \kappa^{2}
$$

To bound $I_{2}$, we use Jensen's inequality and condition (1.1)

$$
\begin{aligned}
I_{2} & \geq c_{1} \int_{0}^{t} \inf _{y \in \mathbf{R}} \mathrm{E}|u(s, y)|^{2(1+\beta)} \int_{\mathbf{R}} p^{2}(t-s, x-y) \mathrm{d} y \mathrm{~d} s \\
& \geq c_{1} \int_{0}^{t}\left(\inf _{y \in \mathbf{R}} \mathrm{E}|u(s, y)|^{2}\right)^{1+\beta} \int_{\mathbf{R}} p^{2}(t-s, x-y) \mathrm{d} y \mathrm{~d} s .
\end{aligned}
$$

Combining the above estimates yields

$$
\mathrm{E}|u(t, x)|^{2} \geq \kappa^{2}+c_{1} \int_{0}^{t}\left(\inf _{y \in \mathbf{R}} \mathrm{E}|u(s, y)|^{2}\right)^{1+\beta} \int_{\mathbf{R}} p^{2}(t-s, x-y) \mathrm{d} y \mathrm{~d} s .
$$

We now use the fact that $\|p(s, \cdot)\|_{L^{2}(\mathbf{R})}^{2} \asymp s^{-1 / \alpha}$ and taking infimum on the left hand side of the above display, we have

$$
\inf _{x \in \mathbf{R}} \mathrm{E}|u(t, x)|^{2} \geq \kappa^{2}+c_{2} \int_{0}^{t}\left(\inf _{y \in \mathbf{R}} \mathrm{E}|u(s, y)|^{2}\right)^{1+\beta} \frac{1}{(t-s)^{1 / \alpha}} \mathrm{d} s .
$$

Upon setting

$$
I(\rho):=\int_{0}^{\infty} e^{-\rho s} \inf _{x \in \mathbf{R}} \mathrm{E}|u(s, x)|^{2} \mathrm{~d} s
$$


for some $\rho>0$, multiplying the above inequality by $e^{-\rho t}$ and then integrating from 0 to $\infty$, we obtain

$$
\begin{aligned}
I(\rho) & \geq \frac{\kappa^{2}}{\rho}+c_{2} \int_{0}^{\infty} \frac{e^{-\rho s}}{s^{1 / \alpha}} \mathrm{d} s \int_{0}^{\infty} e^{-\rho s}\left(\inf _{x \in \mathbf{R}} \mathrm{E}|u(s, x)|^{2}\right)^{1+\beta} \mathrm{d} s \\
& \geq \frac{\kappa^{2}}{\rho}+\frac{c_{3}}{\rho^{1-1 / \alpha}} \int_{0}^{\infty} e^{-\rho s}\left(\inf _{x \in \mathbf{R}} \mathrm{E}|u(s, x)|^{2}\right)^{1+\beta} \mathrm{d} s \\
& \geq \frac{\kappa^{2}}{\rho}+\frac{c_{3} \rho^{\beta+1 / \alpha}}{\rho} I(\rho)^{1+\beta} .
\end{aligned}
$$

We have used Jensen's inequality and some algebra for the last inequality. Hence we have

$$
\rho I(\rho) \geq \kappa^{2}+c_{3} \rho^{1 / \alpha-1}[\rho I(\rho)]^{1+\beta} .
$$

The inequality implies that $I(\rho)$ blows up for some range of $\rho$. We shall show that

$$
I(\rho)=\infty \quad \text { if } \quad 0<\rho \leq\left(c_{3} \kappa^{2 \beta}\right)^{\frac{\alpha}{\alpha-1}} .
$$

Note that under the assumption that $0<\rho \leq\left(c_{3} \kappa^{2 \beta}\right)^{\frac{\alpha}{\alpha-1}}$, the constant $A:=$ $c_{3} \rho^{1 / \alpha-1} \kappa^{2 \beta}$ is strictly bigger than one. We use the fact that $\rho I(\rho) \geq \kappa^{2}$ to reduce the above inequality to

$$
\rho I(\rho) \geq \kappa^{2}+c_{3} \rho^{1 / \alpha-1} \kappa^{2 \beta} \rho I(\rho) .
$$

A simple recursive argument shows that for any positive integer $n$,

$$
\rho I(\rho) \geq \kappa^{2}+A^{n} \rho I(\rho) .
$$

Since $A>1, \kappa>0$ and $n$ is arbitrary. We see that $\rho I(\rho)=\infty$. We have thus shown that $I(\rho)=\infty$ for a range $\rho$. Suppose that there exists a $\rho_{0}<\infty$ which is the minimum of all $\rho$ such that $I(\rho)<\infty$ for all $\rho>\rho_{0}$. But this contradicts (3.5). Hence, there is no finite-energy solution.

The proof of the second part of the theorem will follow the following strategy. We assume that there exists a mild solution satisfying

$$
\sup _{0<s<t-\infty<x<\infty} \sup _{0<1} \mathrm{E}|u(s, x)|^{2}<\infty,
$$

for some $t>0$. If no such $t$ exists, then the theorem is true and there is nothing to prove. In fact, we can take $t$ as large as we wish. We then show that this second moment will blow up in finite time so that (1.6) cannot hold for all $0<T<\infty$ and hence there is no random field solution. We start with the mild formulation of the solution but take expectation to obtain

$$
\mathrm{E} u(t, x)=\int_{\mathbf{R}} u_{0}(y) p(t, x-y) \mathrm{d} y+\int_{0}^{t} \int_{\mathbf{R}} \operatorname{Eb}(u(s, y) p(t-s, x-y) \mathrm{d} y \mathrm{~d} s .
$$

We aim to show that the function $G(s)$ defined by (3.1) blows up in finite time. Proposition 3.1 and an application of Hölder's inequality will then yield the result. We begin by multiplying the above display by $p(t, x)$ and integrate over $\mathbf{R}$ to obtain

$$
\begin{aligned}
G(s) & =\int_{\mathbf{R}} \int_{\mathbf{R}} u_{0}(y) p(t, x-y) \mathrm{d} y p(t, x) \mathrm{d} x \\
& +\int_{\mathbf{R}} \int_{0}^{t} \int_{\mathbf{R}} \operatorname{Eb}(u(s, y)) p(t-s, x-y) \mathrm{d} y \mathrm{~d} s p(t, x) \mathrm{d} x \\
& :=I_{3}+I_{4} .
\end{aligned}
$$


$I_{3}$ is easily seen to be bounded below by $\kappa$. We now bound $I_{4}$. The growth condition on $b$ gives

$$
\begin{aligned}
I_{4} & =\int_{\mathbf{R}} \int_{0}^{t} \int_{\mathbf{R}} \operatorname{Eb}(u(s, y)) p(2 t-s, y) \mathrm{d} y \mathrm{~d} s \\
& \geq c_{5} \int_{\mathbf{R}} \int_{t_{0}}^{t}\left(\frac{s}{2 t-s}\right)^{1 / \alpha} \int_{\mathbf{R}} \mathrm{E}|u(s, y)|^{1+\gamma} p(s, y) \mathrm{d} y \mathrm{~d} s .
\end{aligned}
$$

We now apply Jensen's inequality twice to obtain

$$
\begin{aligned}
I_{4} & \geq c_{5} \int_{t_{0}}^{t}\left(\frac{s}{2 t-s}\right)^{1 / \alpha}\left(\int_{\mathbf{R}} \mathrm{E}|u(s, y)| p(s, y) \mathrm{d} y\right)^{1+\gamma} \mathrm{d} s \\
& \geq c_{5} \int_{t_{0}}^{t}\left(\frac{s}{2 t}\right)^{1 / \alpha} G(s)^{1+\gamma} \mathrm{d} s .
\end{aligned}
$$

Combining the bounds on $I_{3}$ and $I_{4}$, we obtain

$$
G(t) \geq \kappa+c_{5} \int_{t_{0}}^{t} \frac{\left(G(s) s^{1 / \alpha}\right)^{1+\gamma}}{2 t^{1 / \alpha} s^{\gamma / \alpha}} \mathrm{d} s
$$

which in turn yields

$$
G(t) t^{1 / \alpha} \geq \kappa t^{1 / \alpha}+c_{5} \int_{t_{0}}^{t} \frac{\left(G(s) s^{1 / \alpha}\right)^{1+\gamma}}{2 s^{\gamma / \alpha}} \mathrm{d} s \text { for } t \geq t_{0} .
$$

We set $Y(t):=G(t) t^{1 / \alpha}$ and look at the following ODE: $Y^{\prime}(t) t^{\gamma / \alpha}=c_{6} Y(t)^{1+\gamma}$ with $Y\left(t_{0}\right)=\kappa t_{0}^{1 / \alpha}$. Now if $\gamma / \alpha \leq 1$, then $Y(t)$ blows up in finite time, whenever the initial condition satisfies (1.3). But if $\gamma / \alpha>1$, then $Y(t)$ blows up whenever $Y\left(t_{0}\right)$ is large enough. But for any fixed $\kappa$, we can always choose $t_{0}$ large enough so that we have blow up for any $\kappa>0$. Since $G(t)$ blows up whenever $Y(t)$ blows up, the second part of the theorem is proved.

Remark 3.2. In the proof of the second part of the theorem, we have used the fact that $I_{3}$ is bounded below by $\kappa$. One can try to get rid of the assumption that the initial condition is bounded below by using Lemma 2.2. Indeed if we use this lemma with $u_{0}$ instead of $f$, then $G(t) t^{1 / \alpha}$ will be bounded below for large $t$ even if $u_{0}$ is not bounded below. We do not pursue this idea here. We will do so in a forthcoming paper.

\section{REMARKS AND EXTENSIONS}

We start this section by comparing our results with some interesting blow-up results for the stochastic heat equation. Results of Mueller [11,12 and Mueller and Sowers [13] show that when the fractional Laplacian is replaced by the Dirichlet Laplacian on the interval $[0,1]$, then (1.2) has a global solution when $b(x)=0$ and $\sigma(x)=|x|^{\gamma}$ for $1<\gamma<3 / 2$ but the solution blows up whenever $\gamma>3 / 2$. So the critical parameter in this situation is $3 / 2$. The proof of our result suggests that for the Laplacian on the real line this critical parameter is in fact 1.

We end this paper with a couple of possible extensions. Our main result was proved under the assumption that $d=1$. It would be interesting to prove a similar result for $d>1$ but with a noise which has some spatial covariance. One would thus need to find suitable conditions on the spatial covariance as well. This might not 
be an easy task as has been shown in [6] where such equations were studied. One should also note that under Condition [1.2, non-existence is due to additive nonlinearity and the theorem is proved by looking at the expectation of the solution. Hence in this case, one can easily extend the result to higher dimensions as do not need estimates on the relevant stochastic integrals.

Another interesting question is to consider global existence of (1.2), when $b(u)$ plays the role of a damping term, instead of a source term. In this case there will be competition between $b(u)$ and $\sigma(u) \dot{w}(t, x)$, and non-existence will ultimately depend on which term dominates.

\section{ACKNOWLEDGEMENTS}

The authors thank a very careful referee whose comments lead to a more accurate version of this paper.

\section{REFERENCES}

[1] Jonathan M. Blackledge, Application of the fractional diffusion equation for predicting market behaviour, IAENG Int. J. Appl. Math. 40 (2010), no. 3, 130-158. MR2732471(2011h:91229)

[2] René A. Carmona and S. A. Molchanov, Parabolic Anderson problem and intermittency, Mem. Amer. Math. Soc. 108 (1994), no. 518, viii+125, DOI 10.1090/memo/0518. MR.1185878 (94h:35080)

[3] Daniel Conus, Mathew Joseph, and Davar Khoshnevisan, Correlation-length bounds, and estimates for intermittent islands in parabolic SPDEs, Electron. J. Probab. 17 (2012), no. 102, 15, DOI 10.1214/EJP.v17-2429. MR3005720

[4] Robert C. Dalang, Extending the martingale measure stochastic integral with applications to spatially homogeneous s.p.d.e.'s, Electron. J. Probab. 4 (1999), no. 6, 29 pp. (electronic), DOI 10.1214/EJP.v4-43. MR.1684157(2000b:60132)

[5] Mohammud Foondun and Davar Khoshnevisan, Intermittence and nonlinear parabolic stochastic partial differential equations, Electron. J. Probab. 14 (2009), no. 21, 548-568, DOI 10.1214/EJP.v14-614. MR2480553 (2010c:60183)

[6] Mohammud Foondun and Davar Khoshnevisan, On the stochastic heat equation with spatially-colored random forcing, Trans. Amer. Math. Soc. 365 (2013), no. 1, 409-458, DOI 10.1090/S0002-9947-2012-05616-9. MR2984063

[7] O. A. Ladyzhenskaya, The mathematical theory of viscous incompressible flow, Second English edition, revised and enlarged. Translated from the Russian by Richard A. Silverman and John Chu. Mathematics and its Applications, Vol. 2, Gordon and Breach, Science Publishers, New York-London-Paris, 1969. MR0254401 (40 \#7610)

[8] R.S Lakes, Viscoelastic materials, Cambridge University Press, Cambridge, UK, 2009.

[9] Howard A. Levine, The role of critical exponents in blowup theorems, SIAM Rev. 32 (1990), no. 2, 262-288, DOI 10.1137/1032046. MR1056055 (91j:35135)

[10] Keng Deng and Howard A. Levine, The role of critical exponents in blow-up theorems: the sequel, J. Math. Anal. Appl. 243 (2000), no. 1, 85-126, DOI 10.1006/jmaa.1999.6663. MR:1742850(2001b:35031)

[11] Carl Mueller, On the support of solutions to the heat equation with noise, Stochastics Stochastics Rep. 37 (1991), no. 4, 225-245. MR.1149348(93e:60122)

[12] Carl Mueller, The critical parameter for the heat equation with a noise term to blow up in finite time, Ann. Probab. 28 (2000), no. 4, 1735-1746, DOI 10.1214/aop/1019160505. MR.1813841(2002a:60104)

[13] Carl Mueller and Richard Sowers, Blowup for the heat equation with a noise term, Probab. Theory Related Fields 97 (1993), no. 3, 287-320, DOI 10.1007/BF01195068. MR.1245247 (94m:60128)

[14] Ken-iti Sato, Lévy processes and infinitely divisible distributions, Cambridge Studies in Advanced Mathematics, vol. 68, Cambridge University Press, Cambridge, 1999. Translated from the 1990 Japanese original; Revised by the author. MR 1739520 (2003b:60064)

[15] Sadao Sugitani, On nonexistence of global solutions for some nonlinear integral equations, Osaka J. Math. 12 (1975), 45-51. MR0470493 (57 \#10247) 
[16] John B. Walsh, An introduction to stochastic partial differential equations, École d'été de probabilités de Saint-Flour, XIV-1984, Lecture Notes in Math., vol. 1180, Springer, Berlin, 1986, pp. 265-439, DOI 10.1007/BFb0074920. MR876085(88a:60114)

School of Mathematics, Loughborough University, Le11 3TU, United Kingdom \& Mathematics and Computer Science and Engineering division, KAUST, Saudi Arabia

E-mail address: m.i.foondun@lboro.ac.uk

School of Mathematics, Loughborough University, LE11 3TU, United Kingdom \& Mathematics and Computer Science and Engineering division, KAUST, Saudi Arabia

Current address: Department of Mathematics, Clarkson University, Potsdam, NY 13699

E-mail address: Rana.Parshad@kaust.edu.sa 